Research Paper

\title{
ATF4 promotes lung cancer cell proliferation and invasion partially through regulating $\mathrm{Wnt} / \beta$-catenin signaling
}

\author{
Jiang Du¹, Haifeng Liu${ }^{1}$ Xiaoyun Mao², Yanan Qin¹, Chuifeng Fan ${ }^{1 凶}$ \\ 1. Department of Pathology, First affiliated hospital and College of Basic Medical Sciences of China Medical University, 110001, Shenyang, China. \\ 2. Department of Breast Surgery, Department of Surgical Oncology, Research Unit of General Surgery, the First Affiliated Hospital of China Medical \\ University, 110001, Shenyang, China. \\ $\triangle$ Corresponding author: E-mail: cffan@cmu.edu.cn; Tel.: +86 24 23282248; Fax: +86 2423282248.
}

(C) The author(s). This is an open access article distributed under the terms of the Creative Commons Attribution License (https://creativecommons.org/licenses/by/4.0/). See http://ivyspring.com/terms for full terms and conditions.

Received: 2020.12.17; Accepted: 2021.01.07; Published: 2021.01.29

\begin{abstract}
Activating transcription factor 4 (ATF4) is a member of the cAMP response element binding (CREB) protein family and has been reported to participate in cancer progression; however, its molecular mechanism is not fully understood. In this study, we investigated the function of ATF4 in non-small cell lung cancer and its molecular regulation. We detected cytoplasmic and nuclear ATF4 expression in lung cancer A549, H1299, and LK2 cells, and the total expression of ATF4 was higher than that in HBE cells $(p<0.05)$. Higher nuclear ATF4 expression was detected in all these cells compared to cytoplasmic ATF4 expression $(p<0.05)$. Overexpression of ATF4 in A549 cells significantly promoted cancer cell growth and invasion $(p<0.05)$. Expression of Wnt signaling molecules, including $\beta$-catenin, MMP7, and cyclin D1, and the activity of canonical Wnt signaling were also significantly promoted by ATF4 $(p<0.05)$. ICG001, a canonical Wnt signaling inhibitor that selectively inhibits $\beta$-catenin/ cyclic adenosine monophosphate response element binding protein (CBP) interaction, significantly inhibited cancer cell invasion and Wnt signaling. The function of ATF4 was also significantly inhibited by ICG001 $(p<0.05)$. However, compared to treatment with ICG001, the invasion ability of cancer cells treated with both ICG001 and ATF4 CDNA significantly increased $(p<0.05)$, which indicates that the function of ATF4 was not dependent only on Wnt/ $\beta$-catenin signaling. The function of ATF4 in the regulation of $\beta$-catenin expression was not significantly affected by ICG00I ( $p>0.05)$. The function of ATF4 to promote the activity of $\mathrm{Wnt} / \beta$-catenin signaling in cancer cells was abolished by treatment with ICG001 $(p>$ $0.05)$. These results indicate that ATF4 may contribute to lung cancer progression at least partly by regulating $\mathrm{Wnt} / \beta$-catenin signaling.
\end{abstract}

Key words: ATF4; $\beta$-catenin; lung cancer; proliferation; invasion

\section{Introduction}

Lung cancer is one of the most common tumors worldwide [1]. Its pathological types mainly include small cell carcinoma and non-small cell lung carcinoma (NSCLC). The main pathological types of NSCLC are squamous cell carcinoma (SCC) and adenocarcinoma [2]. At present, the main treatment for non-small cell lung cancer is surgery. However, molecular targeted therapy has become an important treatment for patients with advanced NSCLC, which has played a significant role in improving the survival of patients [3, 4]. Understanding the molecular mechanism of lung cancer invasion and metastasis is an indispensable basis for the development of targeted therapy.

The Wnt gene was first identified in mice, called Int-1. It is named Wnt because of its high homology with the wingless gene of Drosophila melanogaster [5, 6]. The Wnt pathway plays an important role in regulating embryonic development and cell differentiation $[5,6]$. Abnormalities in $W n t / \beta$-catenin signaling have been found in many tumors, including NSCLC, and play important roles in cancer progression [7]. Activating transcription factor 4 (ATF4) is a stress response protein induced by hypoxia and other factors [8]. It has been found to regulate cell damage under conditions such as 
hypoxia and chemotherapy [8-10]. ATF4 is also involved in the differentiation of cells, including osteocytes [11]. The role of ATF4 in malignant tumors has been widely reported, but its role is still controversial, which may be related to specific cell types and molecular context. Zeng's study indicates that ATF4 promotes invasion and progression of breast carcinomas [12], and Chen's experiments confirmed that ATF4 promotes proliferation and invasion of gliomas [13]. Others have provided evidence of its anti-cancer function $[9,14]$, and Dai proved that ATF4 inhibits the progression of pancreatic cancer [14]. ATF4 participates in the regulation of many molecular pathways. Zeng proved that ATF4 contributes to the malignant features of breast cancer by regulating E-cadherin [12]. Other molecular links include PERK, MYC, and CCL2 [14-16]. Some experiments show that ATF4 regulates Wnt/ $\beta$-catenin signaling $[11,17]$. However, its role and molecular mechanism in lung cancer cells are still unclear. In this study, we focused on the function of ATF4 and its role in the activation of Wnt/ $\beta$-catenin signaling.

\section{Materials and methods}

\section{Cell culture and transfection}

We used human bronchial epithelial HBE cells and NSCLC A549, H1299, and LK2 cells. HBE, A549, and NCI-H1299 cells were obtained from the American Type Culture Collection (Manassas, VA), and LK2 cells were obtained from the Japanese Collection of Research Bioresources Cell Bank (JCRB). Cells were cultured in RPMI 1640 at $37{ }^{\circ} \mathrm{C}$ in a humidified atmosphere with $5 \% \mathrm{CO}_{2}$. The ATF4 cDNA clone was transfected using Lipofectamine 2000 (Invitrogen, Carlsbad, CA, USA) according to the manufacturer's instructions. The medium was supplemented $12 \mathrm{~h}$ after transfection. G418 was used for post-transfection selection.

\section{Fluorescence microscopy}

Fluorescence microscopy was performed as previously described [18]. Cells were treated with $4 \%$ paraformaldehyde for $30 \mathrm{~min}$ and then washed twice with PBS. The cells were then treated with $0.1 \%$ Triton X-100 for 2 min. After washing twice with PBS, the cells were treated with $1 \%$ BSA. They were then incubated with primary antibody. The antibody for detecting ATF4 expression was purchased from Santa Cruz (sc-22800, USA, 1:500). The secondary antibodies (1:200) were conjugated to FITC. DAPI was used for nuclei counterstaining. Immunofluorescent staining of the cells was performed using an Olympus IX51 fluorescent microscope (Olympus, Tokyo, Japan). Images were captured using a CoolPIX 5400 camera
(Nikon, Japan) fixed in a fluorescent microscope.

\section{Western blotting}

Western blotting was performed as described previously [18]. Protein samples were collected, concentrated, and SDS-PAGE protein loading buffer was added. Samples were analyzed using SDS-PAGE and then transferred to a PVDF membrane. The membrane was immediately placed in the prepared western washing solution and rinsed for 1-2 min. The membrane was then treated with blocking solution, followed by incubation with the first antibody overnight. The next day it was incubated with the second antibody (1:2500) at room temperature for $2 \mathrm{~h}$. The primary antibodies used were: anti-ATF4 (sc-22800, Santa Cruz, USA, 1:200), anti- $\beta$-catenin (sc393501, anti-Santa Cruz, USA, 1:200), anti-cyclin D1 (sc-20044, Santa Cruz, 1:200), anti-MMP7 (sc-515703, Santa Cruz, 1:200), anti-Histone H3(sc-517576, Santa Cruz, 1:500) and anti-GADPH (sc-59540, Santa Cruz, USA, 1:1000). The membrane was treated with enhanced chemiluminescence (ECL) for protein bands, and the signals were detected using a BioImaging System. To determine the relative protein expression in nucleus and cytoplasm separately, nuclear and cytoplasmic protein samples were prepared by using the Nuclear plasma separation Kit (Beyotime, China).

\section{Colony formation assay}

Exponentially growing cells $\left(1 \times 10^{3}\right)$ were suspended after digestion with trypsin, added to a culture dish, and gently shaken to allow the cells to disperse evenly. Cells were cultured in an incubator for 2-3 weeks, and the culture medium was changed according to the $\mathrm{pH}$ value. Colony formation was observed regularly during cell culture, and the culture was terminated when visible clones appeared in the culture dish. After washing and fixation with formaldehyde, the samples were stained with Giemsa for $10 \mathrm{~min}$. The number of clones with more than 50 cells was counted under a microscope.

\section{Matrigel invasion assay}

Matrigel invasion assays were performed as previously described [18]. Cell invasiveness was determined using 24-well Transwell (Corning, NY) and Matrigel (BD Bioscience). Frozen matrigel was thawed at $4{ }^{\circ} \mathrm{C}$ overnight, diluted in serum-free medium (1:3), added $(100 \mu \mathrm{L})$ to the upper chamber, and incubated at $37{ }^{\circ} \mathrm{C}$ for at least $5 \mathrm{~h}$ until the matrigel became solid. A cell suspension in $100 \mu \mathrm{L}$ containing $3 \times 10^{5}$ cells was added into the upper chamber. Medium containing 20\% FBS was added to the lower chamber, and the cells incubated at $37^{\circ} \mathrm{C}$ in 
an incubator for $24 \mathrm{~h}$. The cells were then washed with PBS, fixed with 5\% glutaraldehyde, and stained with $2 \%$ crystal violet. After cleaning, the cells on the upper surface were wiped off with a cotton ball, and those that appeared on the lower surface of the filter were observed under the microscope.

\section{Dual-luciferase assay}

Dual-luciferase assays were performed as described by Zhao [19] and Prévotat [6]. Plasmids with the reporter gene included pGL3-OT expressing sites for a wild-type TCF-4 binding for fluorescence detection and pGL3-OF with mutated TCF-4 binding sites. pRL-TK was used as the control plasmid. A dual-luciferase assay system (Promega, Madison, WI) was used to evaluate the expression of reporter genes. To pre-stimulate the Wnt signaling pathway, 100 $\mathrm{ng} / \mathrm{mL}$ of recombinant human Wnt3a (R\&D Systems) was used.

\section{Statistical analysis}

SPSS software (v22.0, SPSS Inc., Chicago, IL, USA) was used for all data analyses. The Student's t-test was used to compare data from the densitometry analysis. All data from in vitro experiments were expressed as means \pm standard deviation (S.D.) and repeated at least three times. $\mathrm{P}$-values were considered significant when less than 0.05 .

A

ATF4

$\mathrm{GADPH}-\infty$
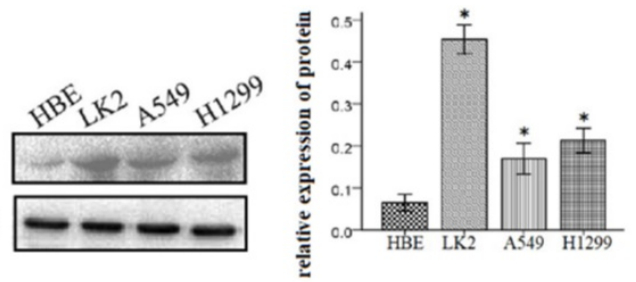

B
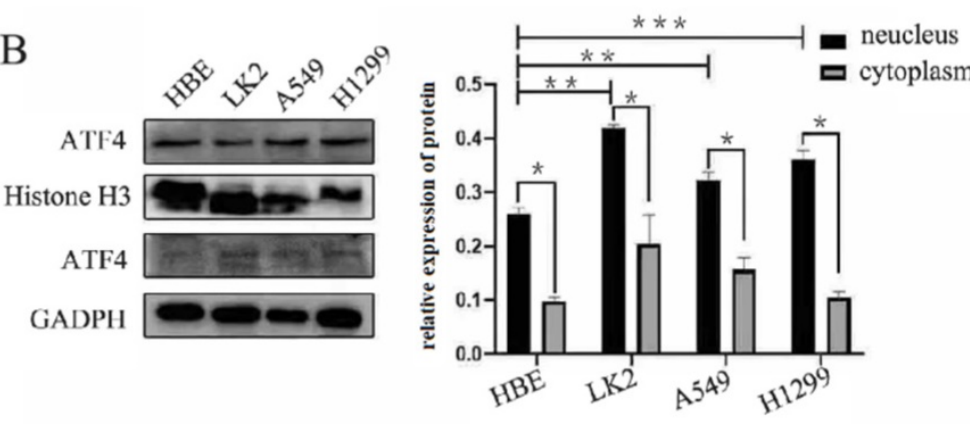

\section{Results}

\section{ATF4 overexpression promotes growth and invasion of A549 lung cancer cells}

We detected ATF4 expression in human bronchial HBE cells and non-small cell lung cancer cells, including A549, H1299, and LK2 cells in vitro using western blotting (Figure 1A and B) and fluorescence microscopy (Figure 1C). Western blotting analysis shows that ATF4 was highly expressed in lung cancer cells compared to HBE cells $(p<0.05)$ (Figure 1A). Nucleus plasma separation experiments showed that ATF4 expression in the nucleus was significantly higher than that in the cytoplasm in all these cells $(p<0.05)$ (Figure 1B). ATF4 expression in cancer cells including A549 and LK2 was significantly higher than that in HBE cells, both in the nucleus and cytoplasm $(p<0.05)$ (Figure 1B). ATF4 expression in nucleus in H1299 cells was significantly higher than that in HBE cells (Figure 1B). To investigate the molecular mechanism underlying the function of ATF4, we selected A549 cells to overexpress ATF4. The results show that ATF4 overexpression significantly promoted cancer cell growth (Figure 2A) and invasion (Figure 2B) $(p<$ $0.05)$. However, ATF4 overexpression in HBE cells did not significantly promote cell invasion in HBE cells $(p$ $>0.05$, Figure $2 \mathrm{C}$ ), which indicates different functions of ATF4 in cancer cells compared with bronchial epithelial cells.

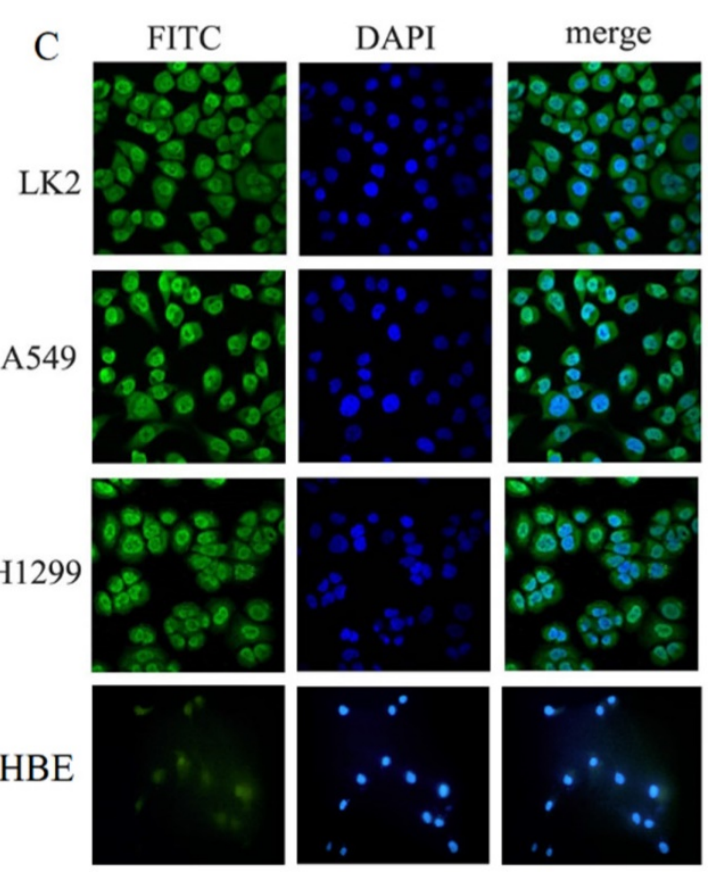

Figure 1. Detection of ATF4 expression in HBE and cancer cells. (A)Western blot study showed an overexpression of ATF4 in cancer cells including A549, H1299 and LK2 compared to normal bronchial epithelial cell HBE $\left({ }^{*} p<0.05\right)$. (B) Nuclear plasma separation experiments showed that ATF4 expression in the nucleus was significantly higher than that in the cytoplasm in cancer cells and HBE cells $\left({ }^{*} p<0.05\right)$. ATF4 expression in A549 and LK2 cells was significantly higher than that in HBE cells, both in the nucleus and cytoplasm (**p < 0.05). ATF4 expression in nucleus in H1299 cells was significantly higher than that in HBE cells (***p < 0.05 ). (C) Fluorescence microscopy also shows both nuclear and cytoplasmic expression of ATF4 in HBE and cancer cells. The experiments were repeated at least 3 times. 


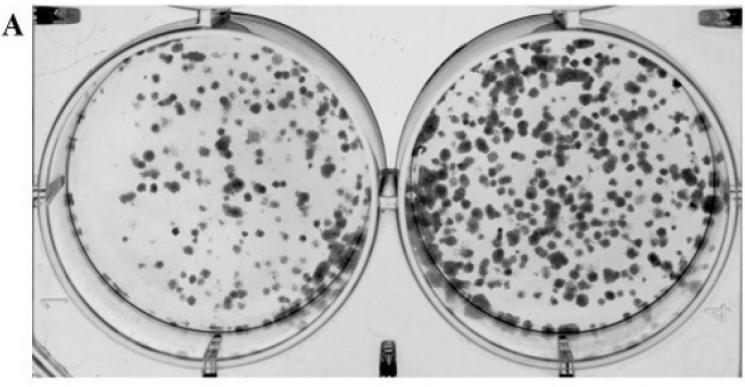

control A549
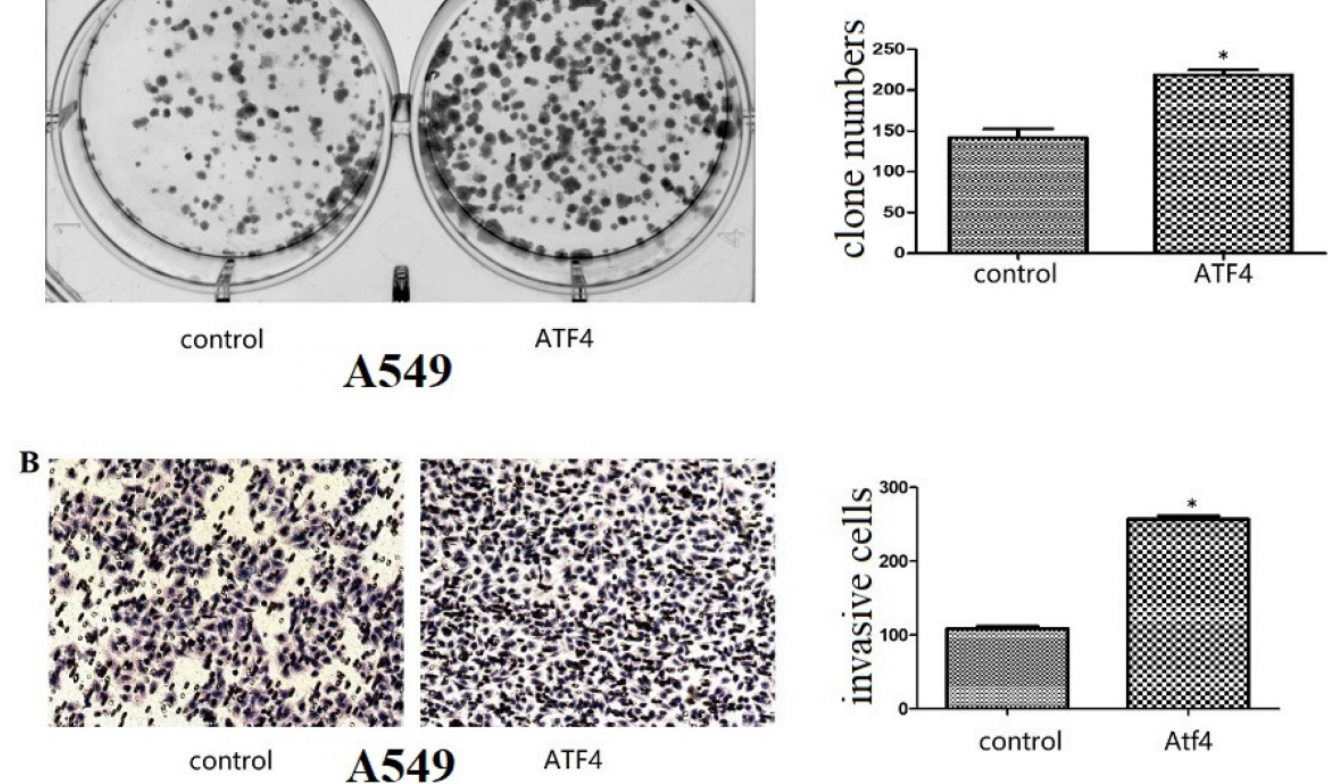

C
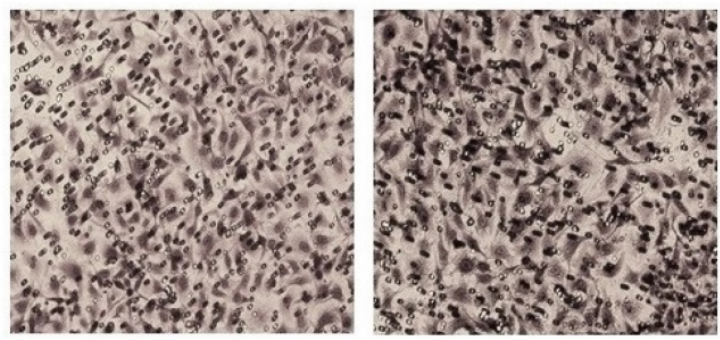

control

HBE

ATF4

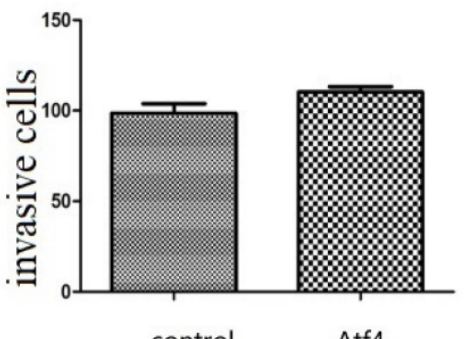

Figure 2. The function of ATF4 to regulate cancer cell biology. (A,B) Overexpression of ATF4 significantly promoted cancer cell growth (A, colony formation study) and invasion (B, Matrigel invasion assay) $(p<0.05)$. (C) ATF4 overexpression in HBE cells did not significantly promote cell invasion $(p>0.05)$. The experiments were repeated at least 3 times. The group of "ATF4" refers to the group with overexpression of ATF4 in A549 cells and HBE cells.

\section{ATF 4 regulates $\beta$-catenin expression and Wnt/ $\beta$-catenin signaling}

Next, we investigated the effects of ATF4 on the Wnt signaling pathway in A549 cells. Western blotting analysis shows that overexpression of ATF4 significantly upregulated the expression of $\beta$-catenin, a key molecule in the canonical Wnt pathway, and of downstream molecules including MMP7 and cyclin D1 $(p<0.05)$ (Figure 3A and 3B). Dual-luciferase assays also show that ATF4 overexpression significantly increased the activity of the canonical Wnt $/ \beta$-catenin signaling pathway $(p<0.05)$ (Figure $3 C)$.

\section{Wnt/ $\beta$-catenin inhibitor ICG00I prevents ATF4 from promoting cancer cell invasion and activating the $W n t / \beta$-catenin signaling pathway}

The Wnt/ $\beta$-catenin inhibitor ICG001 was employed to evaluate the molecular effects of ATF4. ICG001 mainly affects the $\beta$-catenin/CBP interaction and blocks the activation of the canonical Wnt pathway. Matrigel invasion assay shows that ICG001 significantly inhibited cancer cell invasion, whereas ATF4 significantly promoted cancer cell invasion $(p<$ 0.05) (Figure 4). Co-treatment with ICG001 and ATF4, however, significantly inhibited cancer cell invasion $(p<0.05)$ (Figure 4). Compared to treatment with ICG001 alone, the invasion ability of cancer cells was significantly increased by treatment with ICG001 and ATF4 cDNA $(p<0.05)$ (Figure 4$)$, indicating that the invasion-promoting function of ATF4 is not mediated only by Wnt/ $\beta$-catenin signaling.

\section{ATF4-mediated modulation of $\mathrm{Wnt} / \boldsymbol{\beta}$-catenin signaling is abolished by ICG00I}

ICG001 was used to investigate the function of ATF4 in regulating $W n t / \beta$-catenin signaling. ATF4 significantly upregulated $\beta$-catenin expression $(p<$ 
0.05), whereas ICG001 did not significantly change the expression of $\beta$-catenin $(p>0.05)$ (Figure 5). Co-treatment of cells with ATF4 cDNA and ICG001 also significantly upregulated $\beta$-catenin expression $(p$ $<0.05$ ) (Figure 5). Dual-luciferase assays show that both ATF4 and Wnt3a significantly increased the activity of the canonical Wnt pathway ( $\mathrm{p}<0.05)$, whereas ICG001 significantly inhibited its activity with or without Wnt3a $(p<0.05)$ (Figure 6). ICG001 also abolished the function of ATF4 to promote canonical Wnt signaling activation with or without Wnt3a (Figure 6). Compared with treatment with Wnt3a alone, signaling activity did not change significantly in cells treated with both Wnt3a and ATF4 (Figure 6), which indicates that the function of ATF4 may be similar to that of Wnt3a in regulating the stability of the $\beta$-catenin protein.
A

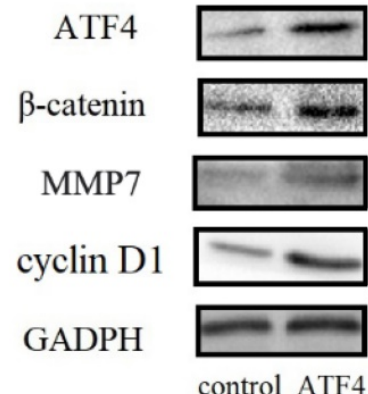

B

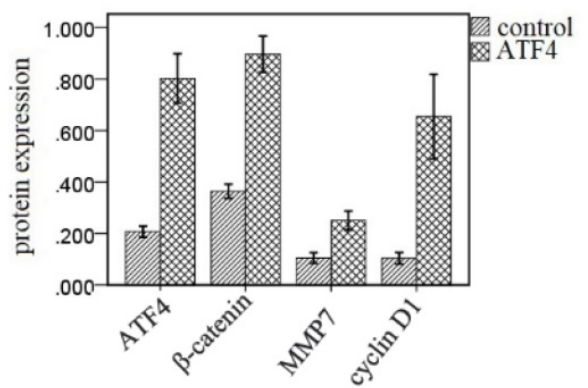

$\mathrm{C}$

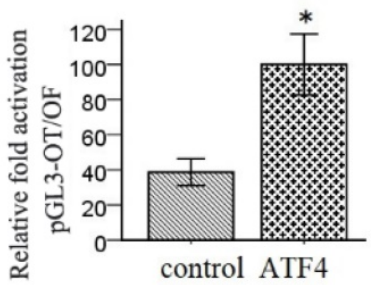

Figure 3. Regulation of Wnt/ $\beta$-catenin signaling by ATF4. (A,B,) Overexpression of ATF4 significantly upregulated the expression of Wnt signaling molecules including $\beta$-catenin, MMP7 and cyclin D1. (C) Dual-luciferase assays showed that overexpression of ATF4 upregulated the activity of Wnt/ $\beta$-catenin signaling ( $p<0.05)$. The group of "ATF4" refers to the group with overexpression of ATF4 in A549 cells. The experiments were repeated at least 3 times.
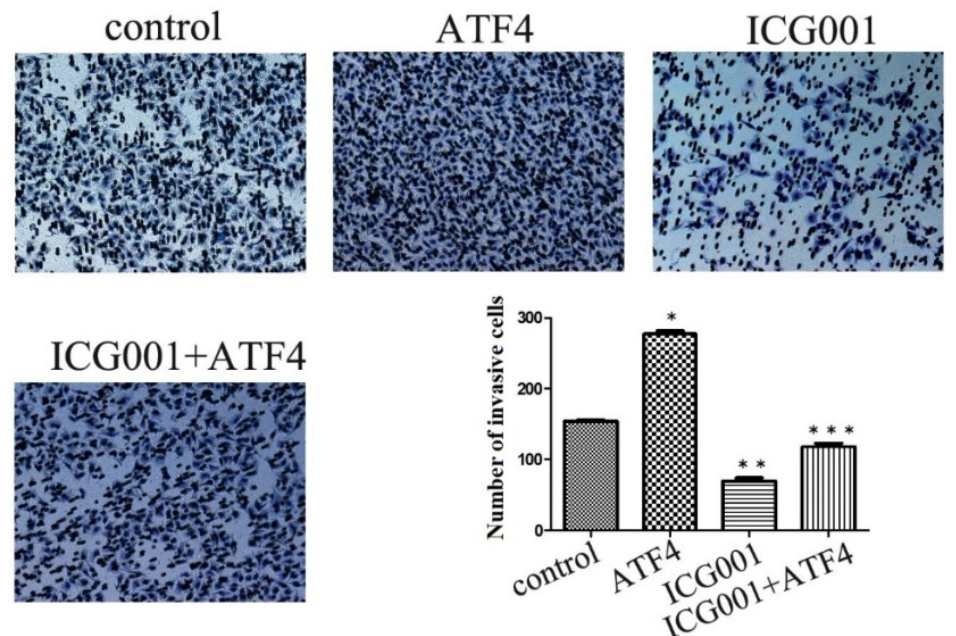

Figure 4. The function of ATF4 to regulate cancer cell invasion was not dependent on Wnt/ $\boldsymbol{\beta}$-catenin signaling. The matrigel invasion assay showed that ATF4 significantly promoted cancer cell invasion $(*, p<0.05)$, while the Wnt/ $\beta$-catenin signaling inhibitor ICG001 inhibited cancer cell invasion $(* *, p<0.05)$. Co-treatment with ICG001 and ATF4, however, significantly inhibited cancer cell invasion $(p<0.05)$. Compared to treatment with ICG001 alone, the invasion ability of cancer cells was significantly increased by treatment with ICG001 and ATF4 CDNA $(p<0.05)$. The experiments were repeated at least 3 times.
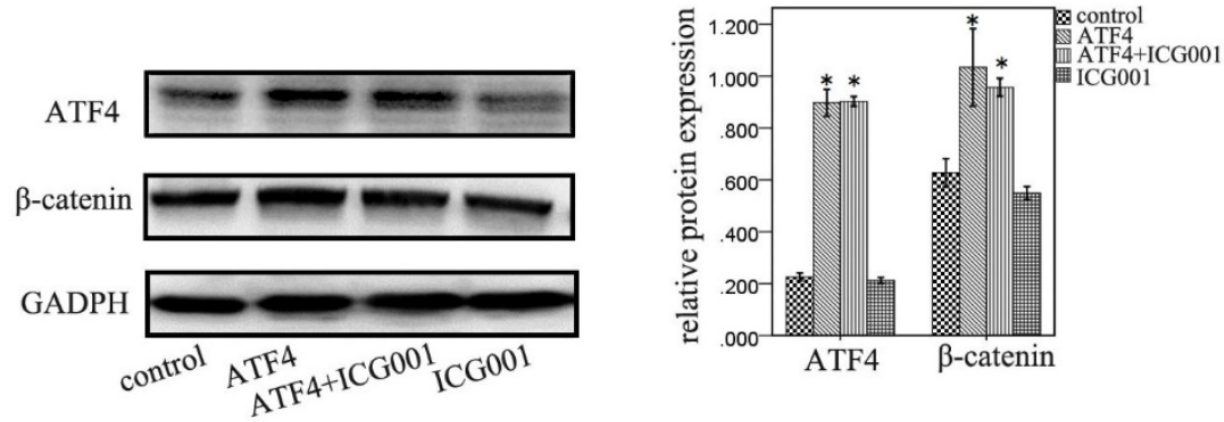

Figure 5. The function of ATF4 and ICG001 to regulate $\beta$-catenin expression. Both ATF4 and co-addition of ATF4 cDNA and ICG001 significantly upregulated $\beta$-catenin expression $(*, p<0.05)$, while treatment with ICG001 only didn't significantly change the expression of $\beta$-catenin $(p>0.05)$. The experiments were repeated at least 3 times. 

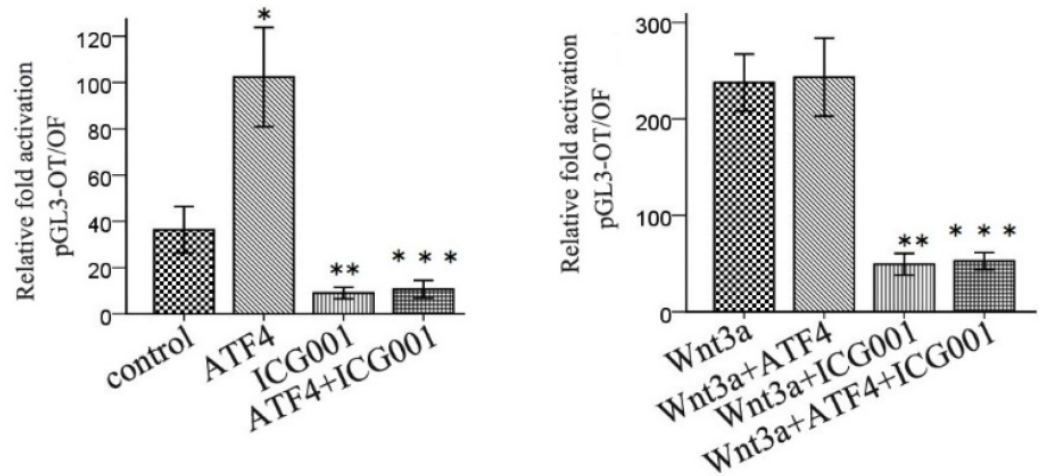

Figure 6. ICG001 abolished the function of ATF4 to regulate the activity of $\mathbf{W n t} / \beta$-catenin signaling. ATF4 significantly increased the activity of $W n t / \beta$-catenin pathway $(*, p<0.05)$, while when treatment with Wnt3a, ATF4 didn't further enhance the activity $(p>0.05)$. ICG001 significantly inhibited the activity with or without Wnt3a $(* *$, $p<0.05)$. ICG001 nearly abolished the function of ATF4 to regulate the activity, with or without Wnt3a $(* * *)$. The experiments were repeated at least 3 times.

\section{Discussion}

ATF4 belongs to the CREB family [12]. It has been shown to be induced by ER stress, oxidative stress, and hypoxia $[8,20]$. Some studies have shown that ATF4 plays an important role in tissue development [21, 22]. Wang et al. showed that ATF4 in the nucleus regulates chondrocyte proliferation and differentiation [22]. In addition, ATF4 was found to be involved in the regulation of metabolism [23]. Here, we investigated ATF4 expression in human bronchial epithelial HBE cells and lung cancer cells. Our data show that ATF4 expression was mainly detected in nucleus in both HBE and cancer cells. In addition, ATF4 expression was upregulated in cancer cells compared to HBE cells in both the nucleus and cytoplasm. However, further studies indicate that there may be different molecular functions of ATF4 in HBE and cancer cells. Our previous study showed that ATF4 is upregulated in NSCLC and may contribute to cancer development. In addition, ATF4 is involved in regulating the biological behavior of tumor cells [24]. In this study, we focused on the molecular links of ATF4 in NSCLC. Lung cancer is one of the most common kinds of cancer and also a leading type of death in cancer [1]. To fully understand the molecular mechanism involved in its progression, it is critical to develop clinical targeted therapies.

ATF4 also has various functions in cancer cells $[11,25]$, although the issue remains controversial. Some studies have indicated the promotion of cancer progression by ATF4 [12, 13, 15-17]. In contrast, some studies have shown that ATF4 inhibits cancer progression $[9,14]$. Participation in the regulation of autophagy by ATF4 was reported [26], and ATF4 also plays a role in sensitivity to chemotherapy $[9,10]$. In the current study, our data indicate that ATF4 had a positive role in promoting cancer growth and invasion. When ATF4 was overexpressed, colony formation increased and invasive cancer cells were more numerous, indicating the important role of ATF4 in regulating lung cancer cell growth and invasion. ATF4 interacts with many molecules and signaling pathways [11-13, 15-17]. Our study showed that ATF4 can upregulate $\beta$-catenin expression and the activity of Wnt/ $\beta$-catenin signaling. To further investigate the underlying molecular mechanism, we used Wnt3a, a ligand that mainly activates canonical Wnt signaling, and the inhibitor ICG001 [27]. The data show that, when cancer cells were treated with Wnt3a, ATF4 did not further enhance the activity of canonical Wnt signaling, which suggests that ATF4 regulates $W n t / \beta$-catenin signaling mainly through modulating $\beta$-catenin stability, similar to Wnt3a. The ICG001 inhibitor mainly affects $W n t / \beta$-catenin signaling by preventing $\beta$-catenin/CBP interaction. In this study, we showed that the function of ATF4 to regulate the $\mathrm{Wnt} / \beta$-catenin pathway was abolished by ICG001, which indicates that ATF4 mainly functions upstream of the $\beta$-catenin/CBP interaction. $\mathrm{Yu}^{\prime} \mathrm{s}$ study showed that ATF4 promotes Wnt/ $\beta$-catenin signaling by inhibiting the degradation of $\beta$-catenin [11], which is consistent with our study. Abnormal activation of $\mathrm{Wnt} / \beta$-catenin signaling plays an important role in many cancers, including NSCLC. Our data indicate that ATF4 may regulate cancer cell growth and invasion at least partly by regulating Wnt/ $\beta$-catenin signaling. MMP7 and cyclin D1 are two critical downstream molecules of Wnt/ $\beta$-catenin signaling, which were also upregulated by ATF4 in this study. MMP7 belongs to the family of matrix metalloproteinases, which play important roles in cancer cell invasion by degrading various protein components of the extracellular matrix, thus destroying the normal histological barrier [28]. Cyclin D1 is one of the cyclins, whose main function is to promote cell proliferation [29]. Further studies to investigate the interaction between ATF4 and $W n t / \beta$-catenin signaling may form the basis for 
the development of targeted therapies. Gao's study indicated that PSAT1 may be the main link between ATF4 and $\beta$-catenin [17]. However, whether PSAT1 contributes to the molecular regulation of Wnt/ $\beta$-catenin signaling by ATF4 in lung cancer cells is not clear. Moreover, our data also showed that the function of ATF4 to regulate cancer cell invasion was not dependent on Wnt/ $\beta$-catenin signaling, and there may be other molecular mechanisms involved. Tameire's study indicated that ATF4 plays an important role in MYC driven tumor progression [16]. The function of ATF4 in regulating cancer progression was also found to be related to PERK and CCL2 $[10,15]$. Whether these molecular links are involved in the function of ATF4 in lung cancer cells needs to be further investigated. In a summary, in this study we found that ATF4 is mainly located in the nucleus of lung cancer cells including A549, LK2 and H1299. ATF4 may promote the growth and invasion of lung cancer cells partly by promoting the activation of canonical Wnt pathway. These results will provide experimental basis for potential clinical targeted therapy.

\section{Abbreviations}

ATF4: activating transcriptional factor 4; CREB: cAMP response element binding; NSCLC: non-small cell lung cancer; CBP: cyclic adenosine monophosphate response element-binding protein; SCC: squamous cell carcinoma.

\section{Acknowledgements}

This work was supported by the Project funded by China Postdoctoral Science Foundation (No. 2019M661171, Jiang Du) and National Natural Science Foundation of China (No. 81472599, Chuifeng Fan).

\section{Competing Interests}

The authors have declared that no competing interest exists.

\section{References}

1. Babar L, Modi P, Anjum F. Lung Cancer Screening. StatPearls. 2020.

2. Travis WD, Brambilla E, Burke AP, Marx A, Nicolson AG. WHO classification of tumors of the lung pleura, thymus and heart. Lyon: IARC. 2015.

3. Lee YT, Tan YJ, Oon CE. Molecular targeted therapy: Treating cancer with specificity. Eur J Pharmacol. 2018; 834:188-96.

4. Chen R, Manochakian R, James L, Azzouqa AG, Shi H, Zhang Y, Zhao Y, Zhou $\mathrm{K}$, Lou $\mathrm{Y}$. Emerging therapeutic agents for advanced non-small cell lung cancer. J Hematol Oncol. 2020; 13(1):58.

5. Wen X, Wu Y, Awadasseid A, Tanaka Y, Zhang W. New Advances in Canonical Wnt/ $\beta$-Catenin Signaling in Cancer. Cancer Manag Res. 2020; 12:6987-6998.

6. Prévotat L, Filomenko R, Solary E, Jeannin JF, Bettaieb A. Nitric oxide-induced down-regulation of beta-catenin in colon cancer cells by a proteasomeindependent specific pathway. Gastroenterology. 2006; 131(4):1142-52.

7. Ng LF, Kaur P, Bunnag N, Suresh J, Sung ICH, Tan QH, Gruber J, Tolwinski NS. WNT Signaling in Disease. Cells. 2019; 8(8).

8. Wortel IMN, van der Meer LT, Kilberg MS, van Leeuwen FN. Surviving Stress: Modulation of ATF4-Mediated Stress Responses in Normal and Malignant Cells. Trends Endocrinol Metab. 2017; 28(11):794-806.
9. Liu J, Qi YB. Activation of LXR $\beta$ inhibits proliferation, promotes apoptosis, and increases chemosensitivity of gastric cancer cells by upregulating the expression of ATF4. J Cell Biochem. 2019; 120(9):14336-47.

10. Shi Z, Yu X, Yuan M, Lv W, Feng T, Bai R, Zhong H. Activation of the PERKATF4 pathway promotes chemo-resistance in colon cancer cells. Sci Rep. 2019; 9(1):3210.

11. Yu S, Zhu K, Lai Y, Zhao Z, Fan J, Im HJ, Chen D, Xiao G. atf4 promotes $\beta$-catenin expression and osteoblastic differentiation of bone marrow mesenchymal stem cells. Int J Biol Sci. 2013; 9(3):256-66.

12. Zeng P, Sun S, Li R, Xiao ZX, Chen H. HER2 Upregulates ATF4 to Promote Cell Migration via Activation of ZEB1 and Downregulation of E-Cadherin. Int J Mol Sci. 2019; 20(9)

13. Chen H, Zhang Y, Su H, Shi H, Xiong Q, Su Z. Overexpression of miR-1283 Inhibits Cell Proliferation and Invasion of Glioma Cells by Targeting ATF4. Oncol Res. 2019; 27(3):325-34.

14. Dai H, Shen K, Yang Y, Su X, Luo Y, Jiang Y, Shuai L, Zheng P, Chen Z, Bie P. PUM1 knockdown prevents tumor progression by activating the PERK/eIF2/ ATF4 signaling pathway in pancreatic adenocarcinoma cells. Cell Death Dis. 2019; 10(8):595.

15. Liu B, Chen P, Xi D, Zhu H, Gao Y. ATF4 regulates CCL2 expression to promote endometrial cancer growth by controlling macrophage infiltration. Exp Cell Res. 2017; 360(2):105-12.

16. Tameire F, Verginadis II, Leli NM, Polte C, Conn CS, Ojha R, Salas Salinas C, Chinga F, Monroy AM, Fu W, Wang P, Kossenkov A, Ye J, Amaravadi RK, Ignatova Z, Fuchs SY, Diehl JA, Ruggero D, Koumenis C. ATF4 couples MYCdependent translational activity to bioenergetic demands during tumour progression. Nat Cell Biol. 2019; 21(7):889-99.

17. Gao S, Ge A, Xu S, You Z, Ning S, Zhao Y, Pang D. PSAT1 is regulated by ATF4 and enhances cell proliferation via the GSK3 $\beta / \beta$-catenin/cyclin D1 signaling pathway in ER-negative breast cancer. J Exp Clin Cancer Res. 2017; 36(1):179.

18. Fan C, Miao Y, Zhang X, Liu D, Jiang G, Lin X, Han Q, Luan L, Xu Z, Wang E. Btbd7 contributes to reduced E-cadherin expression and predicts poor prognosis in non-small cell lung cancer. BMC Cancer. 2014; 14:704.

19. Zhao $Y$, Yang ZQ, Wang $Y$, Miao $Y$, Liu $Y$, Dai SD, Han $Y$, Wang EH. Dishevelled-1 and dishevelled-3 affect cell invasion mainly through canonical and noncanonical Wnt pathway, respectively, and associate with poor prognosis in nonsmall cell lung cancer. Mol Carcinog. 2010;49(8):760-70.

20. Lenna S, Chrobak I, Farina GA, Rodriguez-Pascual F, Lamas S, Lafyatis R, Scorza R, Trojanowska M. HLA-B35 and dsRNA Induce Endothelin-1 via Activation of ATF4 in Human Microvascular Endothelial Cells. PLoS One. 2013; 8(2): e56123.

21. Ritter B, Zschüntsch J, Kvachnina E, Zhang W, Ponimaskin EG. The GABA(B) receptor subunits R1 and R2 interact differentially with the activation transcription factor ATF4 in mouse brain during the postnatal development. Brain Res Dev Brain Res. 2004; 149(1):73-7.

22. Weiguang Wang, Na Lian, Lingzhen Li, Heather E. Moss, Weixi Wang, Daniel S. Perrien, Florent Elefteriou, Xiangli Yang. Atf4 regulates chondrocyte proliferation and differentiation during endochondral ossification by activating Ihh transcription. Development. 2009; 136(24): 4143-53.

23. Seo J, Fortuno ES, Suh J, Stenesen D, Tang W, Parks EJ, Adams CM, Townes T, Graff JM. Atf4 Regulates Obesity, Glucose Homeostasis, and Energy Expenditure. Diabetes. 2009; 58(11): 2565-73

24. Armstrong JL, Flockhart R, Veal GJ, Lovat PE, Redfern CP. Regulation of Endoplasmic Reticulum Stress-induced Cell Death by ATF4 in Neuroectodermal Tumor Cells. J Biol Chem. 2010; 285(9): 6091-100.

25. Singleton DC, Harris AL. Targeting the ATF4 pathway in cancer therapy. Expert Opin Ther Targets. 2012; 16(12):1189-202.

26. Mu N, Lei $Y$, Wang $Y$, Wang $Y$, Duan $\mathrm{O}, \mathrm{Ma}$ G, Liu X, Su L. Inhibition of SIRT1/2 upregulates HSPA5 acetylation and induces pro-survival autophagy via ATF4-DDIT4-mTORC1 axis in human lung cancer cells. Apoptosis. 2019; 24(9-10):798-811.

27. Feng $\mathrm{H}, \mathrm{Hu} \mathrm{H}$, Zheng $\mathrm{P}$, Xun T, Wu S, Yang X, Mo L. AGE receptor 1 silencing enhances advanced oxidative protein product-induced epithelial-tomesenchymal transition of human kidney proximal tubular epithelial cells via RAGE activation. Biochem Biophys Res Commun. 2020; 529(4):1201-8.

28. Shiomi T, Okada Y. MT1-MMP and MMP-7 in invasion and metastasis of human cancers. Cancer Metastasis Rev. 2003; 22(2-3):145-52.

29. Qie S, Diehl JA. Cyclin D1. cancer progression, and opportunities in cancer treatment. J Mol Med (Berl). 2016; 94(12):1313-26. 